\title{
Dealing with Uncertainty in Structured Environments: A Robot Soccer Case Study
}

\author{
Tekin Meriçli, Çetin Meriçli, Ergin Özkucur, \\ Can Kavaklığlu, Barış Gökçe, H. Levent Akın \\ Department of Computer Engineering, Boğaziçi University, \\ Bebek, Istanbul, Turkey \\ E-mail: \{tekin.mericli, cetin.mericli, nezih.ozkucur\}@boun.edu.tr \\ \{can.kavaklioglu, sozbilir, akin\}@boun.edu.tr \\ www.boun.edu.tr
}

\begin{abstract}
Uncertainty is one of the key issues to consider in the field of robotics. Noisy sensor readings and uncontrollable factors in the operation environment as well as the internal mechanisms of the robots may have an accumulating negative effect throughout the decision making process. Starting from the perception of the environment, which is the very first stage of the sense-plan-act cycle, uncertainty should be handled carefully in the localization, world modeling, and action realization stages. In this paper, various techniques we use for dealing with uncertainty in the robot soccer domain are presented and performance comparisons with alternative methods are given.
\end{abstract}

Keywords: uncertainty, world modeling, robot soccer

\section{Introduction}

Physical autonomous robots are expected to operate in real world environments, which are dynamic and most of the time partially observable. RoboCup ${ }^{1}$ offers such environments in its robot soccer leagues where the robots try to solve many real world problems in order to play a higher quality soccer game and beat their opponents. Partial observability combined with noisy sensor readings and actuation leads to uncertainty, and accumulating uncertainty results in unreliable world modeling, which in turn results in wrong decisions. Since the environment is structured, we make use of this prior knowledge throughout the entire process. In this paper, we explain the methods we use as parts of the low level processes to reduce the effect of uncertainty in the higher level decisions as much as possible.

The rest of the paper is organized as follows. The overall system archi- 
tecture is explained in detail in Section 2. Sections 3, 4, and 5 elaborate on the methods used to reduce the effect of uncertainty in several stages of perception, localization, and action realization processes. The paper is summarized and concluded in Section 6.

\section{Controller Architecture}

The overall architecture, shown in Figure 2, is in fact an instance of a classical sense-plan-act paradigm. The process starts with reading sensory information including images from camera, readings of inertial sensors, and current positions of the body joints. Perception module processes the image acquired from camera and perform object recognition. Recognized objects are then used in Self Localization and World Modeling modules incorporates the information about recognized objects and make it ready for the Planner module to make decisions. Appropriate motions corresponding to the decision made by the Planner module are then executed by Motion module.

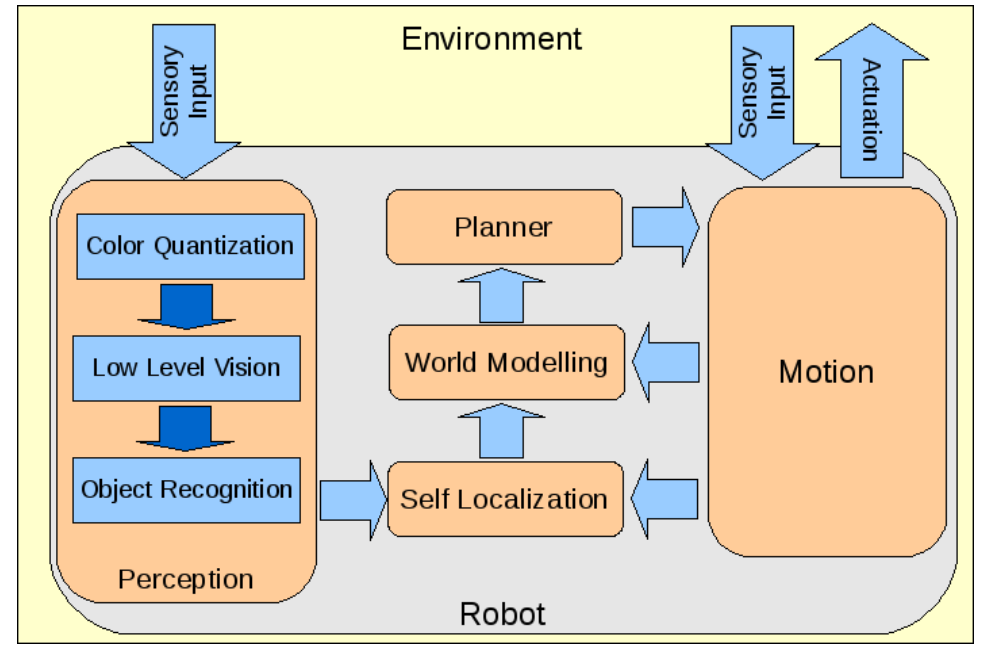

Fig. 1. The overall control architecture of the Cerberus robot soccer team.

\section{Perception}

The entire process starts with perception; that is, grabbing a frame from the camera and processing it to detect the objects on the field. Due to the noise 
associated with the camera and the dynamically changing surrounding of the field, especially at the competition site, false perceptions are always a threat and should be eliminated. In the following sections, we explain the methods we use to deal with uncertainty in several stages of the perception process.

\subsection{Color Segmentation}

As the first step of the perception process, the colors are classified into fewer number of color groups that represent the main colors in the robot soccer environment in order to reduce complexity. This is a regression problem and we used Generalized Regression Neural Network (GRNN) ${ }^{2}$ to solve this problem .

The training sets to be fed to the GRNN are obtained by labeling pixels and regions on some sample images taken from the robots' cameras using our Labeler software. ${ }^{3}$ After a trained network is obtained, a look up table is constructed for all possible inputs in order to reduce the computational complexity of determining the class of a given pixel. Figure 2 shows the output of a trained GRNN on a sample image.

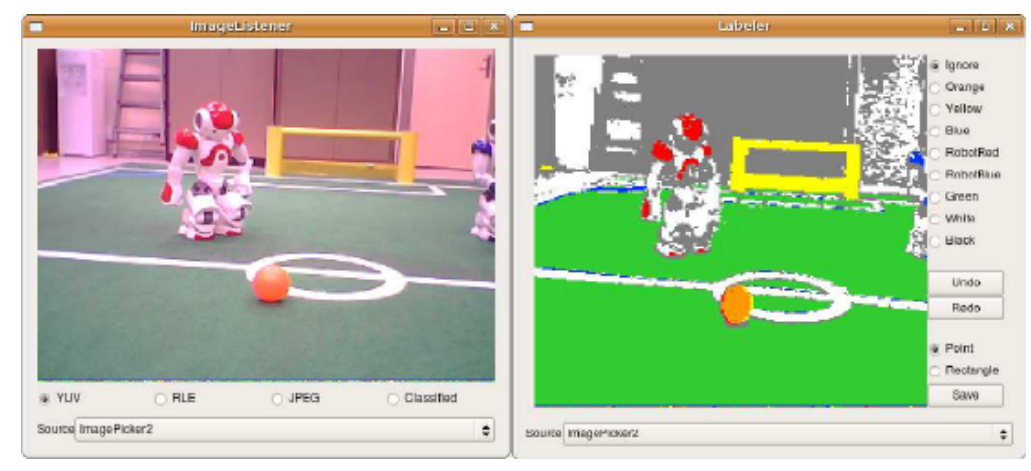

Fig. 2. Result of classification using GRNN.

In addition to GRNN, several experiments are conducted with k-nearest neighbor (k-NN) for the color classification problem. For these experiments, several images of the environment from different point of views are taken from the colored camera of Aibo ERS-7 and labeled manually. These provide a vast dataset for training, validation, and test procedures. First, the dataset is divided into three subsets by random selection, making sure that the subsets contain equal number of images. Then the models are trained 
with the first subset and validated with the second subset for different $\mathrm{k}$ values. Finally, success rates of the trained models are computed using the third subset. Although the success rate of GRNN in classification (92 percent) is not as high as k-NN (98 percent), it is more successful in generalizing the color space, hence reducing the uncertainty in color perception.

\subsection{Perception Pruning}

Although the robot soccer field is a structured environment with known landmark locations, there are many possible combinations of perceived landmark groupings; therefore, sometimes it may be difficult to remove spurious landmarks which can easily seem to be present especially when spectators are present around the field. The most common approach to resolving erroneously perceived landmarks is to use a set of hand coded sanity checking algorithms, which is an incomplete and error prone approach. Due to the very large state space of the visual input, any hand coded static sanity checking algorithm is bound to be invalid for some of the input.

Our approach employs a probabilistic post-perception $(\mathrm{PPM})^{4}$ module to eliminate erroneous detection of landmarks, which only relies on the available map information. This module receives the perceived landmarks from the lower level perception modules and applies domain specific knowledge or previously learned experience to differentiate between the correct and spurious landmarks. The proposed method employs a Hidden Markov Model, ${ }^{2}$ which represents an expectation of landmarks to be perceived. Using this probabilistic expectation, it is possible to detect unexpected landmarks. The results of the experiments conducted indicate that it is possible to generate better results in lower level perception and localization components using the proposed module as can be seen in Figure 3 where the $y$ axis is the cumulative error of the localization engine in millimeters and the $x$ axis is the time in processed camera frames. The blue line indicates the performance of our localization module without PPM and the red line indicates how the PPM affects the localization performance by stabilizing it when misperceptions occur. It can be seen in the figure that eliminating spurious perceptions prevents self localization module to diverge most of the time.

\section{Localization}

Localization module uses two sources of information to determine the pose of the robot on the field: visual perception information and odometry feed- 


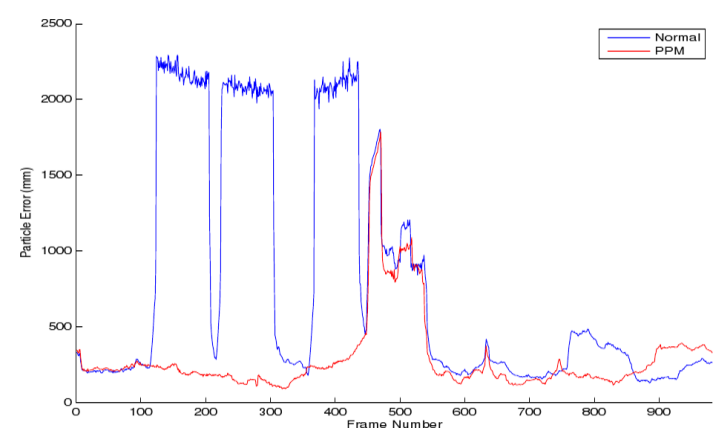

Fig. 3. Cumulative error in self localization estimation over time with (red) and without (blue) PPM.

back. Therefore, keeping the error on these as small as possible has a vital importance for a reliable localization performance.

\subsection{Filtering and Tracking}

The perception module provides instantaneous information. While it is sufficient for reactive behaviors such as tracking the ball with the head, the higher level modules need more information. Especially the planning and localization modules need very accurate perceptual information with reduced noise. Therefore, the world modeling module should reduce the sensor noise and complete the missing information by predicting the state. To solve this problem, we use one of the most common approaches in the literature, which is the Kalman filter. ${ }^{5}$ In Figure 4(a), a snapshot from the filtered world model is given.

Note that despite its small field of view, the robot can remember landmarks from wider range by prediction. With the help of the PPM and the Kalman filter the Monte Carlo Localization method ${ }^{6}$ we use works better and estimates the pose of the robot on the field more accurately. In Figure 4(b), a snapshot from the particle set of the robot is given.

\section{Motion}

The motion module is responsible for realizing the action requested by the planner module. However, the requested action may not always be performed due to obstructions or slippage, resulting in erroneous odometry feedback. In order to reduce the uncertainty in odometry feedback and increase its reliability, these situations should be detected correctly and handled accordingly. 


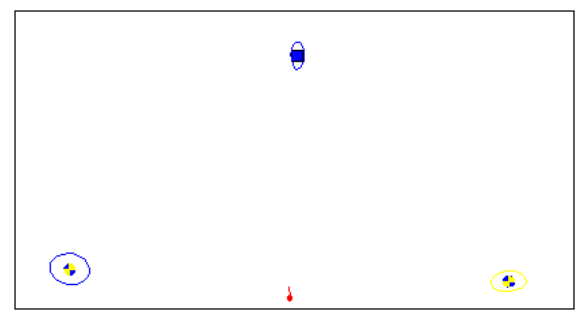

(a) Square represents the goal, circles represent the beacons, and ellipses represent the uncertainty.

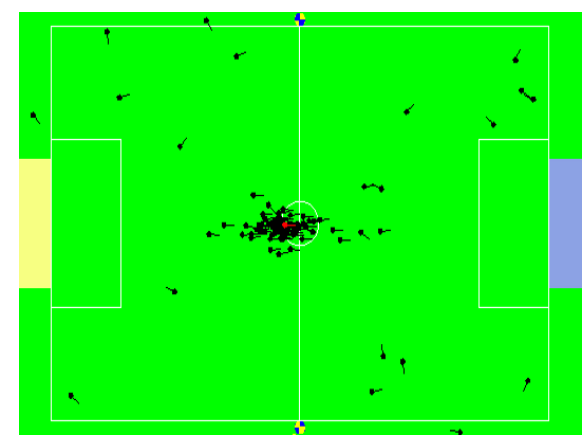

(b) Particle set (belief state) of a robot standing in the middle of the field.

Fig. 4. Snapshot from filtered world model and localization belief state.

\subsection{Collision Detection}

Although the field is flat, collisions and sometimes tumbling are inevitable since there are many robots chasing after a single ball in a rather small field. Figure 5 illustrates one such case where two Aibos collide with each other while chasing the ball.

Detection of and fast recovery from such failures are crucial in robot soccer. Since the $\mathrm{Aibo}^{7}$ and $\mathrm{NaO}^{8}$ robots that we use as our standard platforms are not equipped with specialized touch or bump sensors, we utilized the internal accelerometer readings to decide whether the robot is colliding or about to fall. ${ }^{9}$ Our method considers the problem as an instance of general signal processing and statistical anomaly/novelty detection problem. Since 


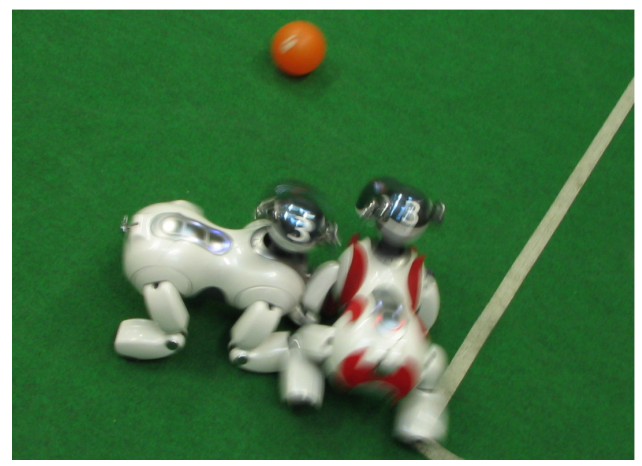

Fig. 5. Two Aibo robots colliding with each other while chasing after the ball.

the walking motion is periodic, the accelerometer readings recorded under normal walking conditions result in a specific dominant frequency value when Fast Fourier Transform is applied to the signal. However, when there is an abnormal situation such as a collision, the dominant frequency is disturbed. Figure 6 illustrates the raw accelerometer signal recorded while the robot is walking forwards and starts colliding with an obstacle at around the $300^{t h}$ frame, and the FFT output for the portions of the signal recorded while the robot is not colliding and colliding, respectively.
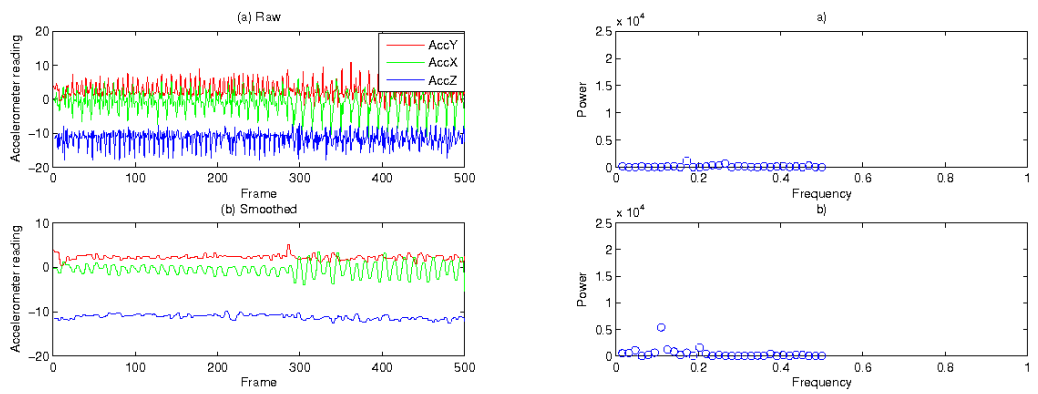

(a) Raw and smoothened accelerometer signal. The robot starts colliding

(b) FFT output for unobstructed with an obstacle at $300^{\text {th }}$ frame.

Fig. 6. (a) Accelerometer readings and (b) frequency / power representation corresponding to the unobstructed and obstructed portions obtained by performing FFT. 
In order to be able to represent the change over time, we defined Sum of Squared Differences (SSD) of two consecutive frequency/power distributions as the sum of squared differences of each power value and its corresponding previous value. Doing that made it possible for us to represent the change in frequency/power distribution between two consecutive frames with a single scalar value. After that, we were able to detect those changes by applying a one tailed t-test to test the hypothesis of whether the observed frequency is coming from the learned distribution of frequencies recorded under normal conditions. When an abnormal situation is detected, the odometry update is suspended in order to prevent the robot from ending up with an incorrect pose estimate and acting improperly. ${ }^{9}$

\section{Conclusion}

Accurate world modeling has a vital importance in decision making in the robot soccer domain. In this paper we present the methods we use as parts of the low level processes to lower the effect of uncertainty in the obtained world model, which include a generalized color classification, perception pruning, filtering and smooth tracking of landmarks, and collision detection for more reliable odometry updates.

\section{References}

1. RoboCup International Robot Soccer Competition http://www.robocup.org.

2. E. Alpaydın, Machine Learning (MIT Press, 2004).

3. H. L. Akın, Ç. Meriçli, T. Meriçli, B. Gökçe, E. Özkucur, C. Kavaklıŏ̆lu and O. T. Ylldiz, Cerberus 2008 RoboCup Standard Platform League Team, tech. rep. (2008).

4. C. Kavaklığlu, Developing a probabilistic post-perception module for mobile robotics, Master's thesis, Boğaziçi University (2009).

5. G. Welch and G. Bishop, An introduction to the Kalman filter, tech. rep. (Chapel Hill, NC, USA, 1995).

6. S. Thrun, D. Fox, W. Burgard and F. Dellaert, Robust monte carlo localization for mobile robots, in Artificial Intelligence, 2001 pp. 99-141.

7. The Sony Aibo robots http://support.sony-europe.com/aibo/.

8. The Nao Humanoid robot http://www.aldebaran-robotics.com/.

9. T. Meriçli, Ç. Meriçli and H. L. Akın, A robust statistical collision detection framework for quadruped robots, in RoboCup International Symposium 2008, (Suzhou, China, 2008). 